Article

\title{
Generation of Hydrogen Peroxide and Downstream Protein Kinase D1 Signaling Is a Common Feature of Inducers of Pancreatic Acinar-to-Ductal Metaplasia
}

\author{
Heike R. Döppler ${ }^{1,+}{ }^{,}$Geou-Yarh Liou ${ }^{1,2,+}$ (D) and Peter Storz ${ }^{1, * \mathbb{D}}$ \\ 1 Department of Cancer Biology, Mayo Clinic, Jacksonville, FL 32224, USA; doeppler.heike@mayo.edu (H.R.D.); \\ gliou@cau.edu (G.-Y.L.) \\ 2 Department of Biological Sciences, Center for Cancer Research \& Therapeutic Development, Clark Atlanta \\ University, Atlanta, GA 30314, USA \\ * Correspondence: storz.peter@mayo.edu; Tel.: +1-904-953-6909; Fax: +1-904-953-0277 \\ + These authors contributed equally to this work.
}

Citation: Döppler, H.R.; Liou, G.-Y.; Storz, P. Generation of Hydrogen Peroxide and Downstream Protein Kinase D1 Signaling Is a Common Feature of Inducers of Pancreatic Acinar-to-Ductal Metaplasia. Antioxidants 2022, 11, 137. https:// doi.org/10.3390/antiox11010137

Academic Editor: Chiara Mozzini

Received: 2 December 2021

Accepted: 6 January 2022

Published: 8 January 2022

Publisher's Note: MDPI stays neutral with regard to jurisdictional claims in published maps and institutional affiliations.

Copyright: (c) 2022 by the authors. Licensee MDPI, Basel, Switzerland. This article is an open access article distributed under the terms and conditions of the Creative Commons Attribution (CC BY) license (https:// creativecommons.org/licenses/by/ $4.0 /)$.

\begin{abstract}
Pancreatic acinar-to-ductal metaplasia (ADM) is a reversible process that occurs after pancreatic injury, but becomes permanent and leads to pancreatic lesions in the presence of an oncogenic mutation in KRAS,. While inflammatory macrophage-secreted chemokines, growth factors that activate epidermal growth factor receptor (EGFR) and oncogenic KRAS have been implicated in the induction of ADM, it is currently unclear whether a common underlying signaling mechanism exists that drives this process. In this study, we show that different inducers of ADM increase levels of hydrogen peroxide, most likely generated at the mitochondria, and upregulate the expression of Protein Kinase D1 (PKD1), a kinase that can be activated by hydrogen peroxide. PKD1 expression in acinar cells affects their survival and mediates ADM, which is in part due to the PKD1 target NF- $\kappa$ B. Overall, our data implicate ROS-PKD1 signaling as a common feature of different inducers of pancreatic ADM.
\end{abstract}

Keywords: hydrogen peroxide; oxidative stress; ADM; acinar-to-ductal metaplasia; pancreas; PKD; Protein Kinase D

\section{Introduction}

In response to inflammation, growth factor signaling or oncogenic signaling, pancreatic acinar cells can undergo a dedifferentiation process named acinar-to-ductal metaplasia (ADM) [1]. ADM is characterized by the transcriptional downregulation of acinar cell markers such as amylase and carboxypeptidase A [2], and increased expression of ductal markers, such as cytokeratin 19 (CK19) and mucin-1 [3]. Cells that undergo ADM are in a less-differentiated, duct-like state $[1,4,5]$, and feature the capacity for pancreatic regeneration. However, ADM cells can be locked into their dedifferentiated state when expressing mutant (active) KRAS. This is an irreversible process, leading to development of low-grade pancreatic lesions, as long as KRAS is active [1,6]. Eventually, low-grade lesions can acquire additional oncogenic mutations and progress to carcinoma in situ and pancreatic cancer [1,5]. Thus, understanding the signaling processes that drive ADM could provide insight into how the pancreas regenerates after a damaging insult, as well as an understanding of the earliest events driving tumor formation.

The signaling mechanisms that regulate ADM can be studied using a three-dimensional (3D) ex vivo organoid cell culture model. In this model, mouse primary acinar cell clusters are isolated from the pancreas, embedded in collagen I matrix and transdifferentiated in the presence of growth factors that activate EGFR (i.e., TGF $\alpha$ and EGF), inflammatory macrophage (IM)-secreted molecules (i.e., TNF, RANTES/CCL5), oncogenic KRAS, or other stimuli [7-11]. These ex vivo studies reliably correlate with animal studies. For 
example, transgenic animal models in which either mutant KRAS [12] or TGF $\alpha$ [13] is expressed in the acinar cells of the pancreas induce acinar cell metaplasia and further progression. Moreover, the induction of pancreatic inflammation with caerulein, a cholecystokinin analog, showed the importance of inflammatory macrophage-secreted factors as drivers of ADM [10]. In this study, we employed this ex vivo 3D cell culture system to investigate the open question of whether different activators of ADM can act though a common signaling pathway.

Using conditional knockout mice, we previously demonstrated that the serine/threonine kinase PKD1 (but not the closely related kinases PKD2 and PKD3) is a critical component of TGF $\alpha$ - and KRAS-driven ADM [14]. Both inducers increase PKD1 expression in vivo and ex vivo in 3D cell culture [14]. Moreover, oncogenic KRAS was shown to induce mitochondrial oxidative stress [15], which is a potent activator of PKD1 [16-18]. Downstream of KRAS, PKD1 has been shown to activate transcriptional regulation via activation of nuclear factor$\kappa B(N F-\kappa B)$ and Notch $[14,15]$, both key factors driving the ADM process $[14,19]$. The aim of the current study was to determine whether the generation of hydrogen peroxide is a critical factor that is common to different activators of ADM. Another aim was to determine whether $\mathrm{H}_{2} \mathrm{O}_{2}$ drives the dedifferentiation process to duct-like cells through the kinase PKD1.

\section{Materials and Methods}

\subsection{Cells, Antibodies and Reagents}

The raw 264.7 macrophage cells (ATCC, Manassas, VA, USA) were maintained in DMEM (high glucose) media containing 10\% FBS and $100 \mathrm{U} / \mathrm{mL}$ penicillin/streptomycin in a $37{ }^{\circ} \mathrm{C}$ incubator supplemented with $5 \% \mathrm{CO}_{2}$. To obtain the conditioned media, $3 \times 10^{6}$ cells per $10 \mathrm{~cm}$ dish were grown in media used for acinar cell 3D collagen explant culture (Waymouth's media plus supplements, see below) and supernatants were collected. The conditioned media was freshly prepared for each experiment. The PKD1 antibody (AP06569PU-N) was from Acris (San Diego, CA, USA), the $\beta$-actin (A5441) antibody was from Sigma-Aldrich (St. Louis, MO, USA). The secondary HRP-linked anti-mouse or anti-rabbit antibodies were from Jackson ImmunoResearch (West Grove, PA, USA). The recombinant murine CCL5/RANTES and TNF $\alpha$ were purchased from PeproTech (Rocky Hill, NJ, USA). The recombinant TGF $\alpha$ was from R\&D Systems (Minneapolis, MN, USA). The hydrogen peroxide was from Thermo Fisher (Rochester, MN, USA) and the EUK134 was from Cayman Chemical (Ann Arbor, MI, USA). The PKD-specific inhibitor kb-NB142-70 was described previously [14,20]. The collagenase I was from Millipore/Sigma (St. Louis, MO, USA). The rat tail collagen I was from BD Biosciences (San Diego, CA, USA).

\subsection{Viral Vectors and Viral Transduction}

The adenovirus to express catalase (rAD-CVM-Cat), the mitochondria-targeted catalase (rAD-CVM-mCat) and the control, empty adenovirus (rAD-Null), were obtained through the University of Iowa Gene Transfer Vector Core. The adenovirus to express super-

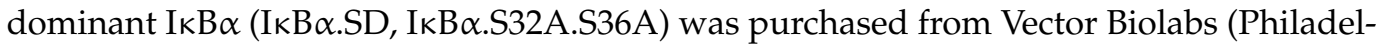
phia, PA, USA). The vectors for the lentiviral expression of PKD1 alleles pLenti6.3/V5GFP-PKD1.CA and pLenti6.3/V5-GFP-PKD1.KD were described previously [14]. The lentiviral plasmids to knock down murine PKD1 were purchased from Sigma-Aldrich. As described in our previous work [14], two hairpin sequences (mPKD1-shRNA\#1 and mPKD1shRNA\#2) showed almost identical efficiency and a specific knockdown of mouse PKD1. Here, we used mPKD1-shRNA\#1 with the following sequence: $5^{\prime}$-CCGGGAGTGTTTGTTGT TATGGAAACTCGAGTTTCCATAACAACAAACACTCTTTTT-3'. The lentiviral plasmids to knock down murine $\mathrm{p} 22^{\text {phox }}$ were purchased from Sigma-Aldrich and are described in [15]. The adenoviral and lentiviral transductions were performed as described in previous research $[14,21]$. In all the experiments, the virus was used at $10^{7} \mathrm{ifu} / \mathrm{mL}$. 


\subsection{Proliferation Assays}

The cells were isolated and infected as indicated. The cells were plated and their DNA content was measured using the CyQUANT Cell Proliferation Assay kit (Invitrogen, Waltham, MA, USA) on a Synergy HT plate reader (BioTek, Winooski, VT, USA).

\subsection{Isolation of Primary Peritoneal Macrophages}

The mice were injected with $2 \mathrm{~mL}$ of $5 \%$ aged thioglycollate. Five days later, the mice were euthanized and the peritoneal macrophages were collected via a single injection of $10 \mathrm{~mL}$ RPMI-1640 plus 10\% FBS into the peritoneal cavity and subsequent withdrawal (described in detail in $[10,22])$. The peritoneal exudate was centrifuged $(233 \times g)$ and washed with RPMI-1640 media plus 10\% FBS before plating onto $10 \mathrm{~cm}$ tissue culture dishes. After one hour at $37^{\circ} \mathrm{C}$, the plates were washed 2-3 times to remove non-adherent cells, and fresh media was added. The conditioned media was prepared as described above for the Raw 264.7 cells. These experiments were conducted under a Mayo Clinic IACUC-approved protocol (A00004882) and were in accordance with institutional guidelines and regulations.

\subsection{Isolation of Primary Pancreatic Acinar Cells}

The primary pancreatic acinar cells were isolated from the C57BL/6J mice using a protocol that was described in detail previously [11,14,21]. In brief, the pancreas was washed twice with ice-cold HBSS media, minced into 1 to $5 \mathrm{~mm}$ pieces and digested with $5 \mathrm{~mL}$ of $2 \mathrm{mg} / \mathrm{mL}$ collagenase I in HBSS $\left(37^{\circ} \mathrm{C}\right.$, shaker). The digestion was terminated by the addition of an equal volume of ice-cold HBSS media with 5\% FBS. The digested pieces were washed twice (HBSS plus 5\% FBS) and pipetted through $500 \mu \mathrm{m}$ and $105 \mu \mathrm{m}$ meshes. The acinar cell suspension was added dropwise to $20 \mathrm{~mL}$ The HBSS media was supplemented with $30 \%$ FBS. The acinar cells were pelleted $\left(233 \times g, 2 \mathrm{~min}, 4{ }^{\circ} \mathrm{C}\right)$ and re-suspended in $10 \mathrm{~mL}$ Waymouth's complete media (1\% FBS, $0.1 \mathrm{mg} / \mathrm{mL}$ trypsin inhibitor, $1 \mu \mathrm{g} / \mathrm{mL}$ dexamethasone). The isolation of the acinar cells was conducted using excess mice and the procedures were performed after the animals were euthanized. All the experiments were in accordance with IACUC regulations.

\subsection{Acinar-to-Ductal Metaplasia Assay}

This method is demonstrated in detail in [21]. In short, freshly isolated primary pancreatic acinar cells were embedded in collagen I/Waymouth's media (w/o supplements). Waymouth's complete media was added on top of the cell/gel mixture and replaced every other day. Inhibitors or compounds were added at indicated concentrations to both the cell/gel mixture and the media on top. For the viral transductions, the acinar cells were infected for 3-5 h before embedding in the collagen I/Waymouth's media mixture. If not indicated otherwise, the number of ducts per well was determined at day 5 , and photographs were taken to document the cellular structures.

\subsection{Measurement of ROS Generation}

The primary pancreatic acinar cells were labeled with $\mathrm{H}_{2}$ DFFDA $(20 \mu \mathrm{M})$ in phenol red-free Waymouth's complete media at $37^{\circ} \mathrm{C}$ for $20 \mathrm{~min}$. The cells then were washed with HBSS, transferred to phenol red-free Waymouth's media and stimulated as indicated. ROS generation (formation of fluorescent DCF) over indicated time was determined at 495/529 nm (excitation/emission) using a SpectraMax M5 fluorescent microplate reader (Molecular Devices, Sunnyvale, CA, USA).

\subsection{Quantitative PCR}

The RNA was extracted using an RNeasy Plus Kit (Qiagen, Germantown, MD, USA), and converted to cDNA using a High Capacity cDNA RT Kit (Applied Biosystems, Foster City, CA, USA). Each qPCR reaction used Taqman Fast 2x PCR Mix (Applied Biosystems) along with mGapdh (Mm99999915_g1), mPrkd1 (Mm00435790_m1), mCyba (p22 ${ }^{\text {phox }}$; Mm00514478_m1), mPdx1 (Mm00435565_m1), mKrt19 (Mm00492980_m1), mHes1, 
(Mm01342805_m1) or mMuc1 (Mm00449604_m1) primer/probe sets (Applied Biosystems). The reactions were run on a QuantStudio 7 Flex Real-Time PCR System (Applied Biosystems). All the $C_{T}$ values were normalized to Gapdh and the $\Delta \Delta C_{T}$ method was used to calculate the fold changes.

\subsection{Cell Lysis and Western Blot}

The cells were washed twice using ice-cold PBS (140 mM NaCl, $2.7 \mathrm{mM} \mathrm{KCl}, 8 \mathrm{mM}$ $\mathrm{Na} 2 \mathrm{HPO} 4$, and $1.5 \mathrm{mM} \mathrm{KH} 2 \mathrm{PO} 4$ [pH 7.2]) and lysed using buffer A (50 mM Tris- $\mathrm{HCl}, \mathrm{pH}$ 7.4, $1 \%$ Triton X-100, $150 \mathrm{mM} \mathrm{NaCl}, 5 \mathrm{mM}$ EDTA, $\mathrm{pH}$ 7.4) plus a protease inhibitor cocktail (Sigma-Aldrich, St. Louis, MO, USA). After a $30 \mathrm{~min}$ incubation on ice, the lysates were centrifuged $\left(16,200 \times g, 4{ }^{\circ} \mathrm{C}, 15 \mathrm{~min}\right)$, the supernatants were collected and the protein concentration was measured using a BioRad Protein Assay (BioRad, Hercules, CA, USA). Following SDS-PAGE $(10 \%$ gel; $30 \mathrm{~mA})$ and transfer to nitrocellulose membrane, proteins of interest were detected via Western blot using the indicated primary antibodies at 1:2000 and horseradish peroxidase (HRP)-conjugated secondary antibodies at 1:5000.

\subsection{Quantification and Statistical Analysis}

All the cell biological and biochemical experiments were performed independently of each other at least three times. For the ADM assays, three biological replicates were performed using pancreata from three different mice. The quantification of the ductal area was performed using ImageJ. The data, when presented as bar graphs, show individual values (dots), mean and \pm standard deviation (SD). If not stated otherwise in the figure legends, the $p$ values were acquired with the unpaired Student's $t$-test with Welch's correction using Graph Pad software (GraphPad Inc., La Jolla, CA, USA). The $p$ values are included in the graphs and $p<0.05$ was considered statistically significant.

\section{Results}

\subsection{TGFa Induces Pancreatic Acinar-to-Ductal Metaplasia through Hydrogen Peroxide}

The activation of EGFR signaling through its ligands, EGF and TGF $\alpha$, in the acinar cells induces their metaplasia to a duct-like phenotype [23], but the underlying cellular signaling is not well understood. The signaling mechanisms driving ADM can be investigated in acinar organoid culture [24]. When seeded in three-dimensional (3D) collagen culture, isolated murine primary acinar cell clusters undergo ADM after treatment with TGF $\alpha$ (Figure 1A). This is accompanied by the upregulation of the mRNA expression of cytokeratin 19 (Krt19), pancreatic and duodenal homeobox 1 (Pdx1), glycoprotein mucin 1 (Muc1), and the Notch target gene Hes1 (Hes1), all typical markers that indicate the transdifferentiation process (Supplemental Figure S1A). We noticed that the treatment of isolated acinar cells with TGF $\alpha$ leads to a slight but steady and significant increase in reactive oxygen species over time (Figure 1B). Such TGF $\alpha$-generated oxidative stress is important for driving the ADM process, since acinar cell organoids that are treated with TGF $\alpha$ in the presence of the antioxidant N-Acetyl-L-Cysteine (NAC), or with EUK134, a synthetic salen-manganese complex with SOD and catalase activity [25], completely fail to undergo transdifferentiation (Supplemental Figure S1B and Figure 1C). Similar inhibitory effects on TGF $\alpha$-induced ADM are observed when acinar cells are transduced with catalase, suggesting that hydrogen peroxide is the oxidative stress component that drives the transdifferentiation process (Figure 1D). 
A
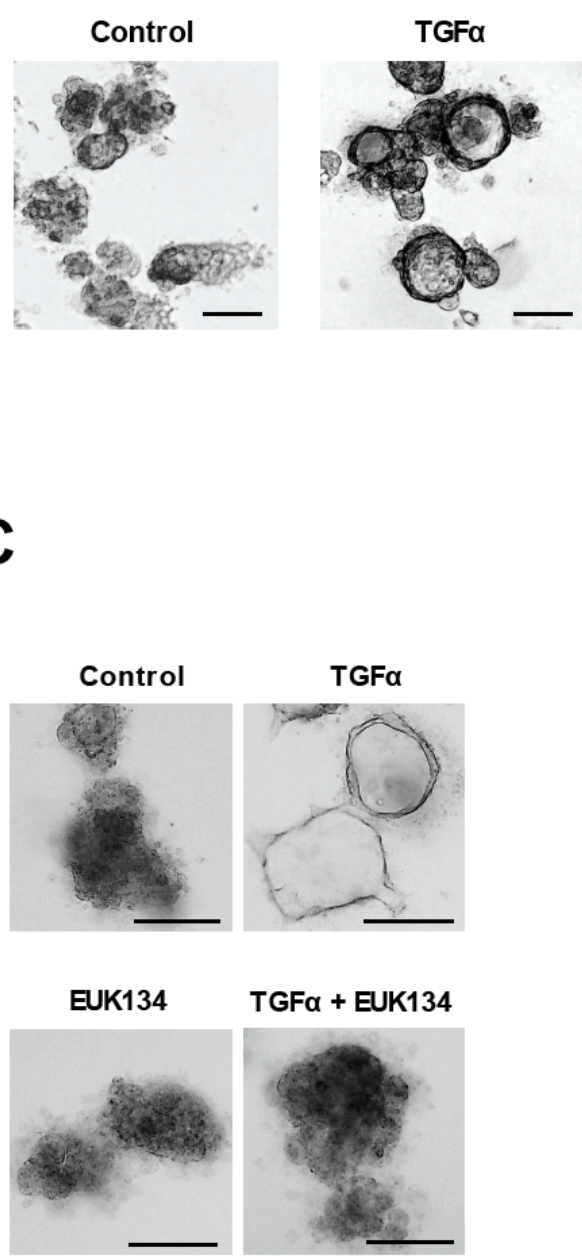

D

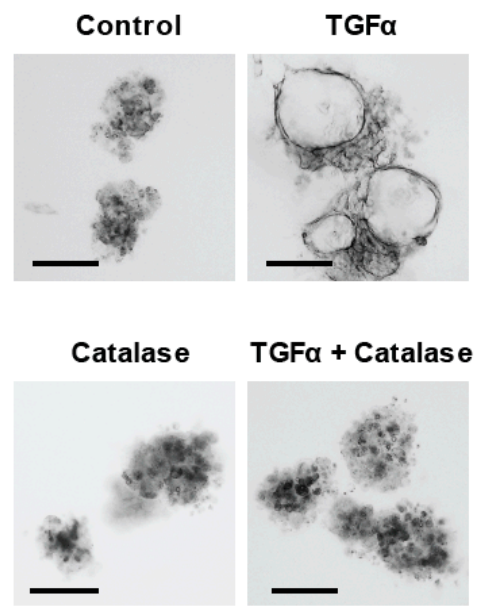

B
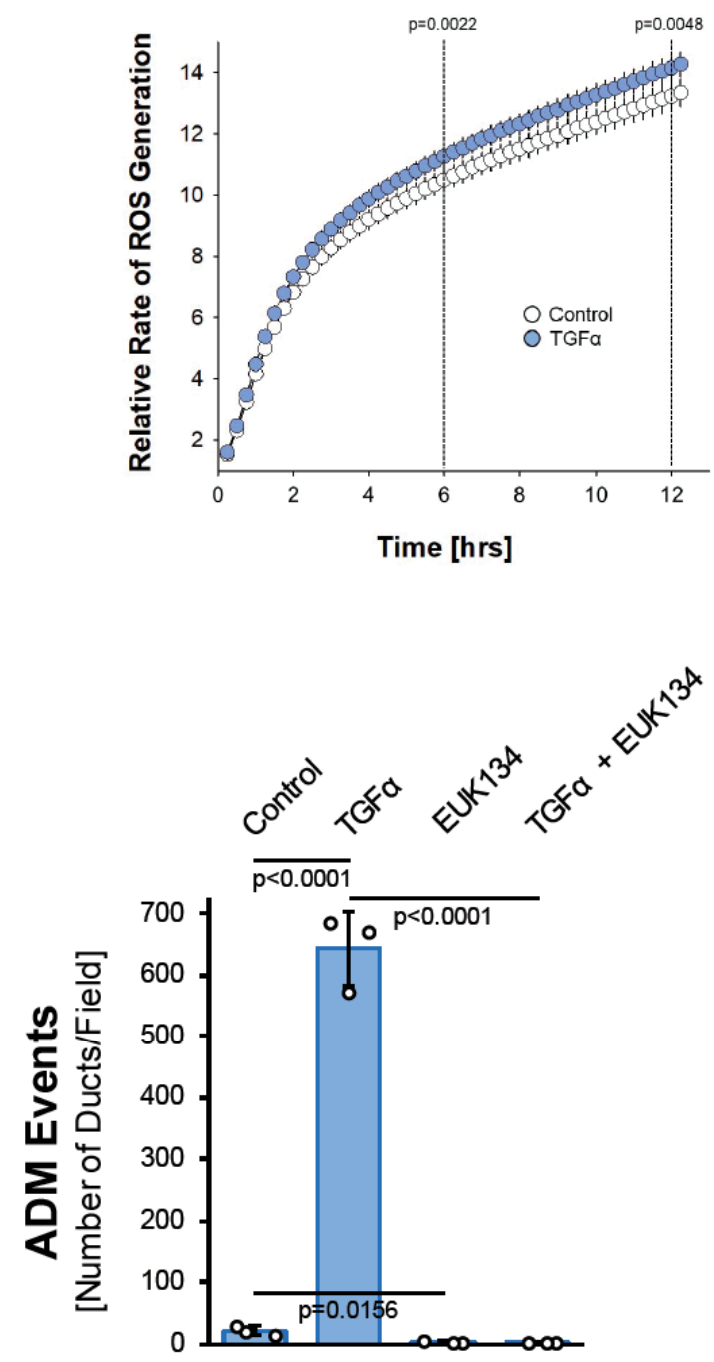

Figure 1. TGF $\alpha$ induces pancreatic acinar-to-ductal metaplasia through hydrogen peroxide. (A): Mouse pancreatic acinar cells were isolated and seeded in 3D collagen culture. ADM was induced with TGF $\alpha(50 \mathrm{ng} / \mathrm{mL})$ and ducts formed were photographed. The bar indicates $100 \mu \mathrm{m}$. (B): Primary 
mouse acinar cells were stimulated as indicated and labeled with H2DFFDA. Generation of intracellular ROS (fluorescent DCF) was measured over the indicated time period. (C): Mouse pancreatic acinar cells were isolated and seeded in 3D collagen culture. As indicated, cells were treated with EUK134 $(25 \mu \mathrm{M})$ and ADM was induced with TGF $\alpha(50 \mathrm{ng} / \mathrm{mL})$. Ducts formed were photographed (left side; bar $=100 \mu \mathrm{m}$ ) and quantified (right side) as indicated in the Methods section. (D): Mouse pancreatic acinar cells were isolated, infected with empty adenovirus (rAD-Null) or adenovirus to express catalase (rAD-CVM-Cat) and seeded in 3D collagen culture. As indicated, ADM was induced with TGF $\alpha(50 \mathrm{ng} / \mathrm{mL})$. Ducts formed were photographed (left side; bar $=100 \mu \mathrm{m})$ and quantified (right side) as indicated in the Methods section. (A-D): All experiments shown were performed in triplicates (except $(B) ; n=6$ ) for at least three times and obtained similar results in each repeat. Statistical analysis between two groups was performed using the Student's $t$-test. A $p$ value of 0.05 was considered statistically significant and values are included in the graphs.

\subsection{Macrophage-Secreted Factors Also Induce Pancreatic ADM through Hydrogen Peroxide}

We next tested whether oxidative stress is a common feature of how different factors drive ADM. We previously showed that macrophage-conditioned media can initiate the ADM process ([10] and Supplemental Figure S2). Similar to what was observed for TGF $\alpha$ (Figure 1B), macrophage-conditioned media also increased cellular oxidative stress (Figure 2A). Moreover, either treatment with EUK134 (Figure 2B) or the transduction of catalase significantly decreased macrophage-conditioned media-induced ADM events (Figure 2C), and ductal area (Figure 2D).

We previously identified the cytokines/chemokines CCL5/RANTES and TNF $\alpha$ as the major factors that drive ADM in macrophage-conditioned media [10], with CCL5 being the more potent inducer (Figure 2D). Both factors increased cellular oxidative stress (Figure 2F). Moreover, as seen for the macrophage-conditioned media, the induction of ADM by both of these factors was efficiently blocked by the transduction of catalase (Figure 2G,H). Overall, these data suggest that the induction of oxidative stress is a common feature downstream of the different inducers of ADM, and the generation of hydrogen peroxide may be a driver of the process.

\subsection{Mitochondria-Generated Hydrogen Peroxide Can Drive Acinar-to-Ductal Metaplasia}

We next tested whether hydrogen peroxide can drive ADM and whether it stems from the NADH/NADPH oxidase complex or from mitochondria. The treatment of acinar organoids with hydrogen peroxide induced ADM approximately twofold (Figure 3A), although the ducts formed were smaller in size when compared to TGF $\alpha$ (Figure 3B). We then tested whether TGF $\alpha$ induces ADM through the hydrogen peroxide that is produced by the NADH/NADPH oxidase complex. A knockdown of p22 ${ }^{\text {phox }}$ (Supplemental Figure S3A), which is a critical component of each of the different NADH/NADPH oxidase complexes, did not affect TGF $\alpha$-mediated ADM (Supplemental Figure S3B). By contrast, the expression of mitochondria-targeted catalase (mitoCat) via adenoviral transduction completely blocked TGF $\alpha$-mediated ADM, suggesting the mitochondrial electron transport chain as a source for hydrogen peroxide driving the transdifferentiation process (Figure 3C). Similarly, ADM induced by M-CM was blocked when a mitochondria-targeted catalase was expressed (Figure 3D), but not when p22 $2^{\text {phox }}$ was knocked down (Supplemental Figure S3C).

\subsection{Hydrogen Peroxide Drives ADM via Protein Kinase D1}

PKD1 has been shown to be activated by hydrogen peroxide [17,18]. Moreover, we previously showed that PKD1 is a key driver of ADM downstream of EGFR and Kras G12D signaling in vitro and in vivo $[14,15]$. Therefore, we next tested whether PKD1 also acts downstream of M-CM and CCL5, making it a common feature needed for the ADM process. In acinar cells, M-CM increased PKD1 levels (Figure 4A) and a knockdown of PKD1, using a previously published specific shRNA [14], decreased the number of ADM events (Figure 4B, Supplemental Figure S4A). Moreover, CCL5, the major factor in M-CM that 
drives ADM, also induced PKD1 expression at mRNA and protein levels (Supplemental Figure S4B, Figure 4C). As observed for M-CM, a knockdown of PKD1 not only decreased the number of CCL5 induced ADM events (Figure 4D), but also the area of ductal structures (Supplemental Figure S4C). Similar effects of PKD1 knockdown have been demonstrated for TGF $\alpha$ and mutant KRAS [14].

A

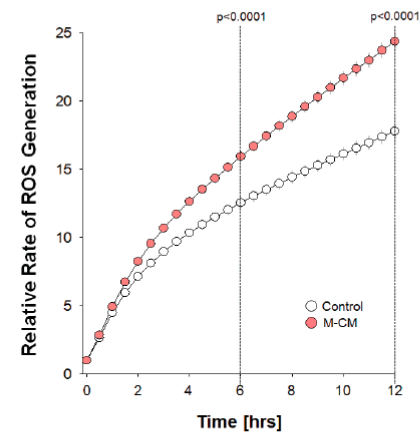

D

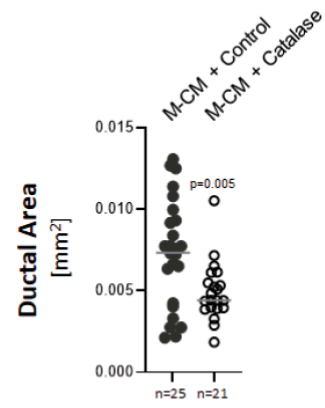

G

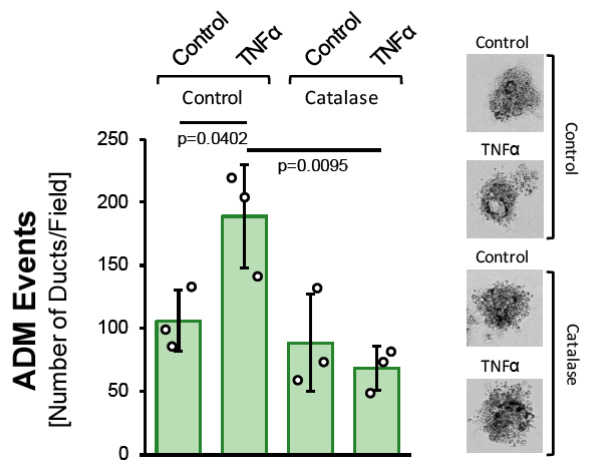

B

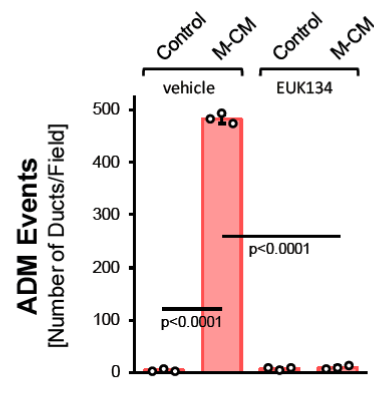

C

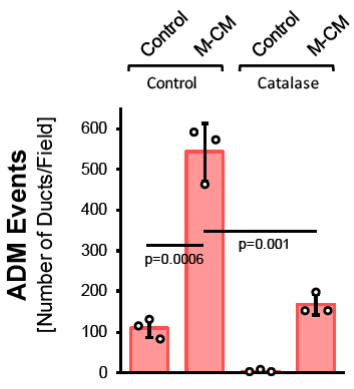

E

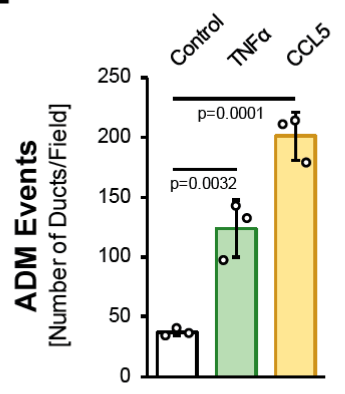

F

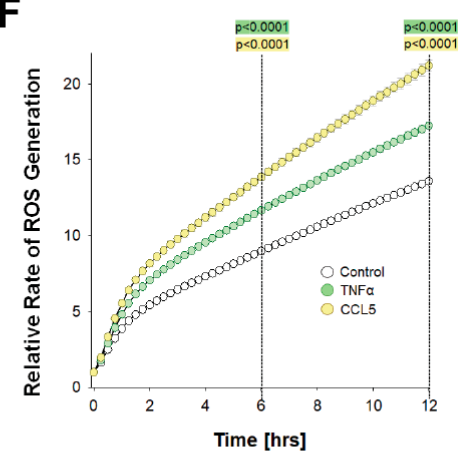

H

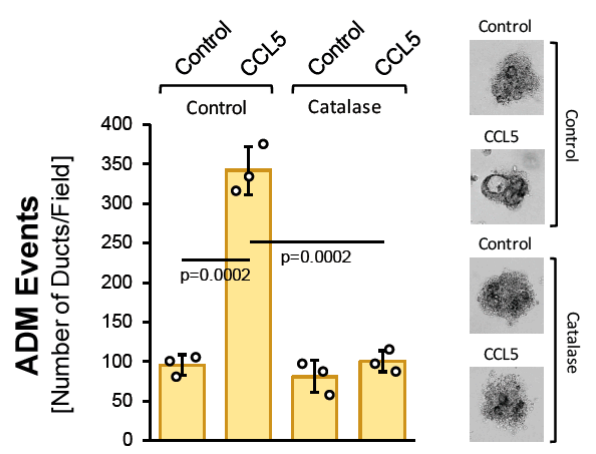

Figure 2. Macrophage-secreted factors induce pancreatic ADM through hydrogen peroxide. (A): Primary mouse acinar cells were stimulated as indicated and labeled with H2DFFDA. Generation of intracellular ROS (fluorescent DCF) was measured over the indicated time period. (B): Mouse pancreatic acinar cells were isolated and seeded in 3D collagen culture. As indicated, cells were treated with EUK134 $(25 \mu \mathrm{M})$ and ADM was induced by replacing the overlaying media with macrophage media (Control) or macrophage-conditioned media (M-CM). Ducts formed were quantified as described in the Methods section. (C): Mouse pancreatic acinar cells were isolated, infected with rAD-Null or rAD-CVM-Cat and seeded in 3D collagen culture. As indicated, effects on ADM were quantified after stimulation with control media or M-CM. (D): Ducts formed after induction of ADM with M-CM following adenoviral expression of catalase (or control) were analyzed for their ductal area using ImageJ. (E): Mouse pancreatic acinar cells were isolated, seeded in 3D collagen culture and stimulated 
with vehicle (Control), TNF $\alpha$ (50 ng/mL) or CCL5 (50 ng/mL). Ducts formed were quantified as indicated in the Methods section. (F): Primary mouse acinar cells were stimulated as indicated and labeled with H2DFFDA. Generation of intracellular ROS (fluorescent DCF) was measured over the indicated time period. $(\mathbf{G}, \mathbf{H})$ : Mouse pancreatic acinar cells were isolated, infected with $\mathrm{rAD}-\mathrm{Null}$ or $\mathrm{rAD}-\mathrm{CVM}$-Cat and seeded in 3D collagen culture. ADM was induced with $50 \mathrm{ng} / \mathrm{mL}$ TNF $\alpha$ (G) or $50 \mathrm{ng} / \mathrm{mL} \mathrm{CCL5} \mathrm{(H)} \mathrm{and} \mathrm{ducts} \mathrm{formed} \mathrm{were} \mathrm{quantified} \mathrm{as} \mathrm{indicated} \mathrm{in} \mathrm{the} \mathrm{Methods} \mathrm{section.}$ Representative pictures are shown on the right side. (A-H): All experiments shown were performed in triplicates for at least three times and obtained similar results in each repeat. Statistical analysis between two groups was performed using the Student's $t$-test. A $p$ value of 0.05 was considered statistically significant and values are included in the graphs.

A
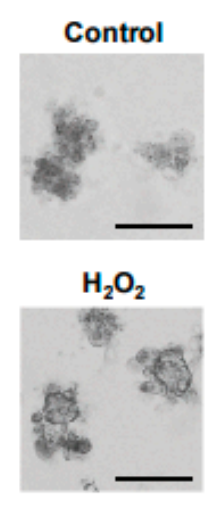

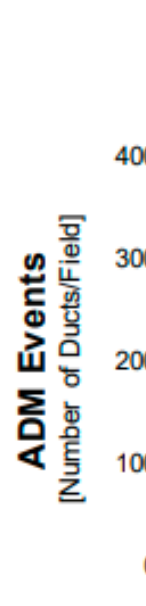<smiles>C1CCC1</smiles>

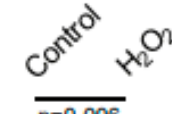
$\overline{\mathrm{p}=0.006}$
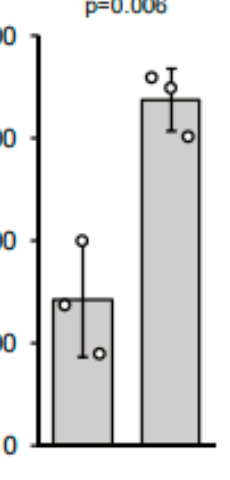

B

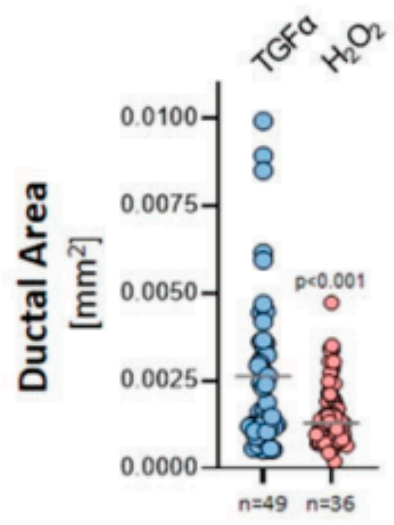

C

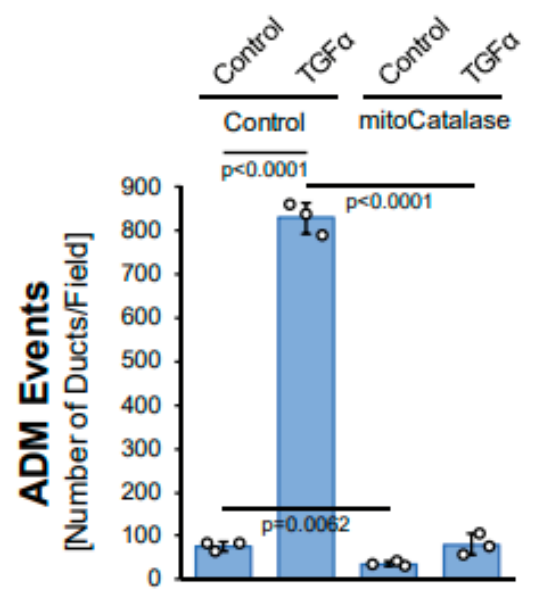

D

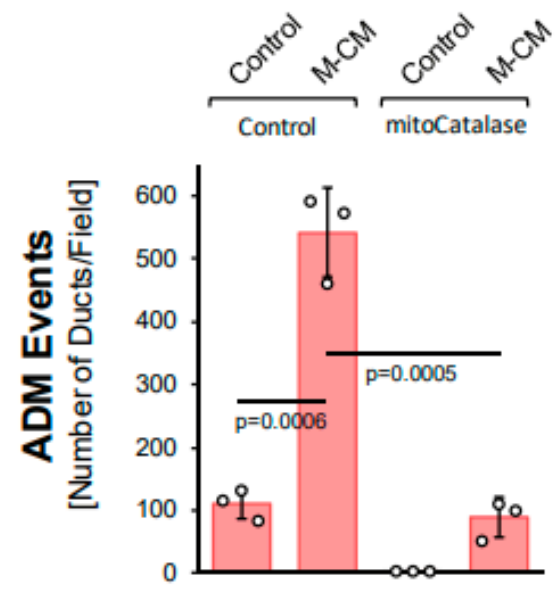

Figure 3. Mitochondria-generated hydrogen peroxide can drive acinar-to-ductal metaplasia. (A): Mouse pancreatic acinar cells were isolated, seeded in 3D collagen culture and stimulated with vehicle (Control) or hydrogen peroxide $\left(\mathrm{H}_{2} \mathrm{O}_{2} ; 10 \mu \mathrm{M}\right)$. Ducts formed were photographed (left side; bar $=125 \mu \mathrm{M})$ and quantified as indicated in the Methods section. (B): Ducts formed after induction of ADM with TGF $\alpha(50 \mathrm{ng} / \mathrm{mL})$ or $\mathrm{H}_{2} \mathrm{O}_{2}(10 \mu \mathrm{M})$ were analyzed for their ductal area using ImageJ. (C,D): Mouse pancreatic acinar cells were isolated, infected with rAD-Null or rAD-CVM$\mathrm{mCat}$ and seeded in 3D collagen culture. ADM was induced with $50 \mathrm{ng} / \mathrm{mL}$ TGF $\alpha(C)$ or M-CM (D) and ducts formed were quantified as indicated in the Methods section. (A-D): All experiments shown were performed in triplicates for at least three times and obtained similar results in each replicate. Statistical analysis between two groups was performed using the Student's $t$-test. A $p$ value of 0.05 was considered statistically significant and values are included in the graphs. 
A

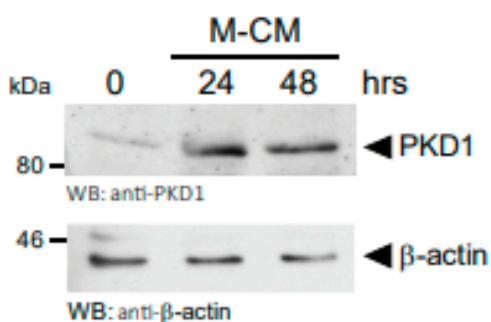

C

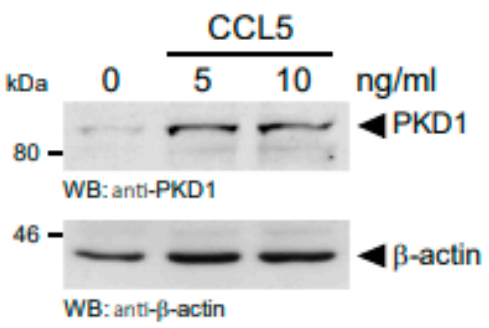

$\mathbf{E}$

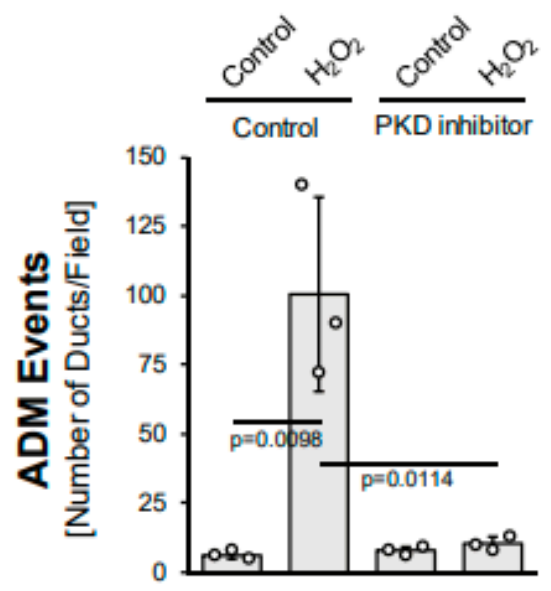

B

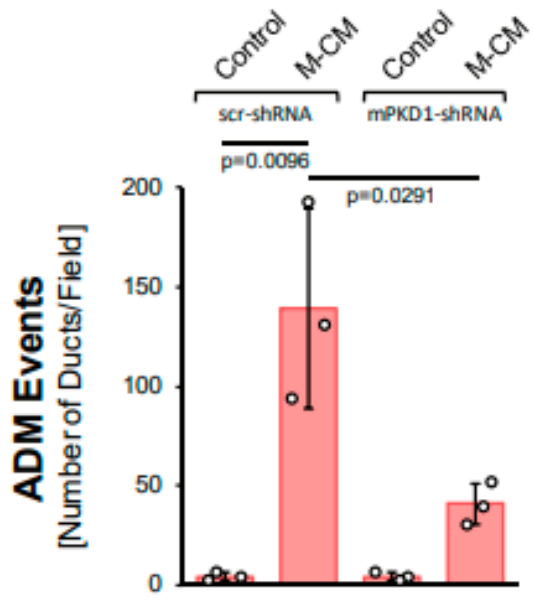

D
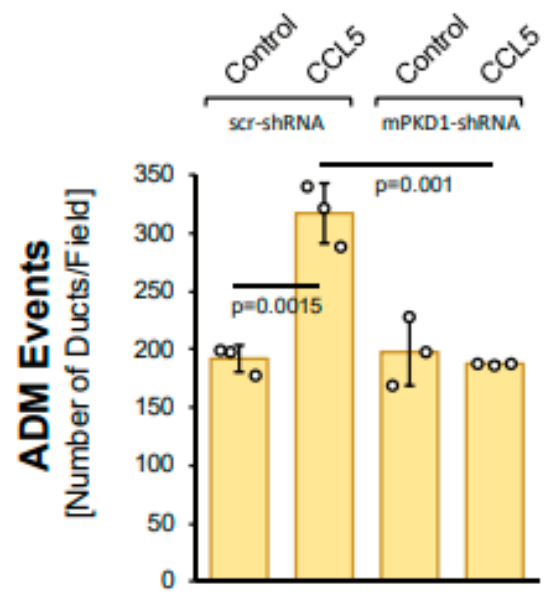

$\mathbf{F}$

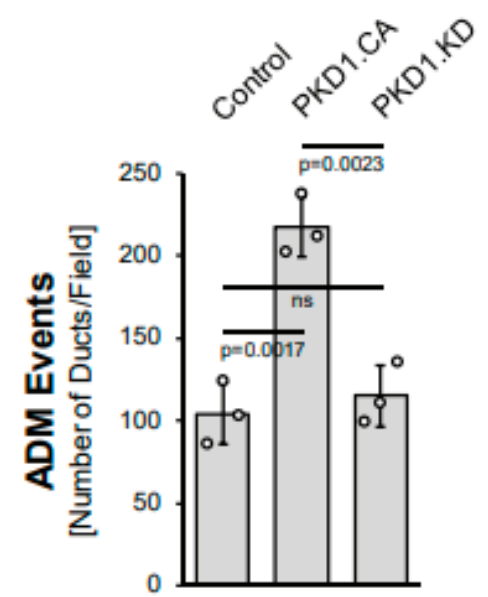

Figure 4. Protein Kinase D1 is downstream of different inducers of ADM. (A): Mouse pancreatic acinar cells were isolated and stimulated with $\mathrm{M}-\mathrm{CM}$ for the indicated time. Cells were lysed and analyzed by Western blot for expression of PKD1 or for $\beta$-actin (control for equal loading). (B): Mouse pancreatic acinar cells were isolated, lentivirally-infected with control (scr-shRNA) or mPKD1-shRNA and seeded in 3D collagen culture. As indicated, ADM was induced by replacing the overlaying media with macrophage media (control) or macrophage-conditioned media (M-CM). Ducts formed 
were quantified as described in the Methods section. (C): Mouse pancreatic acinar cells were isolated and stimulated with CCL5 at indicated dosages for $48 \mathrm{~h}$. Cells were lysed and analyzed by Western blot for expression of PKD1 or for $\beta$-actin (control for equal loading). (D): Mouse pancreatic acinar cells were isolated, lentivirally-infected with control (scr-shRNA) or mPKD1-shRNA, seeded in 3D collagen culture and stimulated with vehicle (Control) or CCL5 (50 ng/mL). Ducts formed were quantified as indicated in the Methods section. (E): Mouse pancreatic acinar cells were isolated, seeded in 3D collagen culture, treated with vehicle (Control) or PKD inhibitor (kb-NB-142-70, $1 \mu \mathrm{M}$ ) and stimulated with vehicle (Control) or hydrogen peroxide $\left(\mathrm{H}_{2} \mathrm{O}_{2} ; 10 \mu \mathrm{M}\right)$, as indicated. Ducts formed in three replicates were quantified as indicated in the Methods section. (F): Mouse pancreatic acinar cells were isolated, lentivirally-infected with either control vector, GFP-tagged PKD1.CA or PKD1.KW and seeded in 3D collagen culture. Ducts formed in three replicates were quantified as indicated in the Methods section. (A-F): All experiments shown were performed in triplicates for at least three times and obtained similar results in each replicate. Statistical analysis between two groups was performed using the Student's $t$-test. A $p$ value of 0.05 was considered statistically significant and values are included in the graphs.

Since hydrogen peroxide is downstream of the inducers of ADM, we then tested whether the induction of ADM via hydrogen peroxide is inhibited when PKD1 activation is blocked. The treatment of acinar cell organoids with the PKD-specific inhibitor kb-NB-14270 showed that active PKD drives ADM downstream of hydrogen peroxide (Figure 4E). Similarly, the lentiviral transduction of a constitutively-active (PKD1.S738E.S742E; PKD1.CA), but not a kinase-dead (PKD1.K612W; PKD1.KD) version of PKD1 into acinar cells led to an increase in ADM events (Figure 4F). Overall, these data suggest the upregulation and ROS-mediated activation of PKD1 as common features essential for the ADM process.

\subsection{PKD1 Drives ADM through Canonical NF- $\kappa B$ Signaling}

We next investigated whether the expression of PKD1 contributes to acinar cell survival. Isolated primary acinar cells, when seeded without surrounding extracellular matrix, undergo apoptosis within a few days [26]. We found that the lentiviral expression of PKD1 in isolated acinar cells can increase acinar cell survival (Figure 5A). Survival downstream of PKD1 is regulated via signaling to one of its main target pathways, canonical NF- $\mathrm{B}$ [18]. NF-kB is also one of the transcription factors that drives ADM [4,15]. Therefore, we tested whether the activation of NF-kB through PKD1 drives ADM in pancreatic acinar cells in 3D explant organoid culture. We found that PKD1-induced ADM can be partially blocked after the adenoviral transduction of a superdominant $\mathrm{I} \kappa \mathrm{B} \alpha(\mathrm{I} \kappa \mathrm{B} \alpha . \mathrm{SD})$, which is mutated to block canonical NF- $\kappa$ B activation (Figure 5B). Similarly, the expression of I $\kappa$ B $\alpha$.SD blocked ADM induced by the PKD1 upstream activators TGF $\alpha$, CCL5 (both Supplemental Figure S5), TNF, MCM (both [10]) and mutant KRAS [15]. This suggests that PKD1 drives ADM not only via the previously reported effects on Notch signaling [14], but also though NF- $\mathrm{B}$. In summary, we show here that ROS-PKD1-NF- $\kappa B$ signaling is downstream of common inducers of acinar cell metaplasia and is a critical driver of the transdifferentiation process. 
A

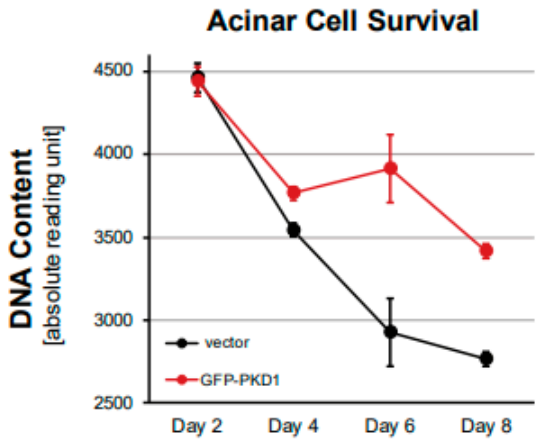

B
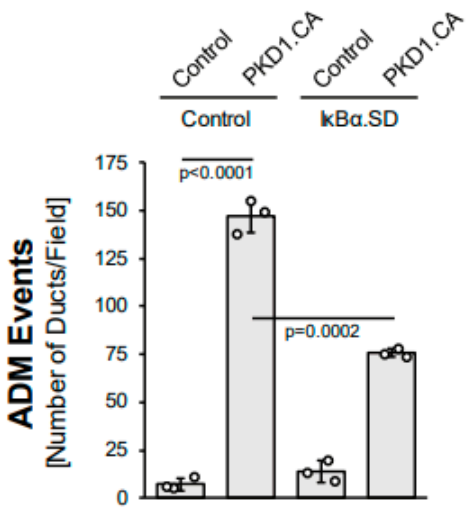

C

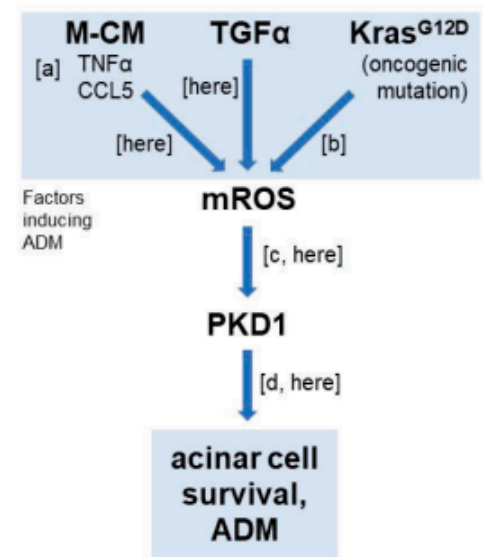

Figure 5. PKD1 increases acinar cell survival and drives ADM through canonical NF- $\mathrm{KB}$ signaling. (A): Primary mouse acinar cells were isolated, lentivirally-infected with control vector or GFP-tagged PKD1, and then subjected to a CyQUANT Cell Proliferation Assay kit over the indicated time period. (B): Mouse pancreatic acinar cells were isolated, adenovirally-infected with control virus

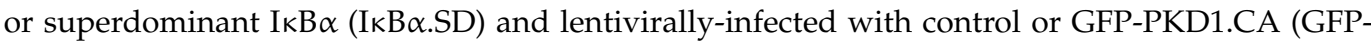
PKD1.SSEE) and then seeded in 3D collagen culture. ADM and ducts formed were quantified as described in the Methods section. (C): Schematic summarizing current data and previously published work, demonstrating how ROS-PKD1 signaling is a key factor downstream of different inducers of acinar-to-ductal (ADM) metaplasia. Macrophage-conditioned medium (M-CM) with its two major drivers TNF $\alpha$ and CCL5 (published in [10]; (a)), TGF $\alpha$ (here), or expression of oncogenic KRAS (published in [15]; (b)) all induce mitochondrial reactive oxygen species (mROS), which have been shown to activate PKD1 (published in [17]; [c] and (here)). PKD1 is a key driver of ADM (published in [14]; (d) and (here)). 


\section{Discussion}

Pancreatic acinar-to-ductal metaplasia occurs in response to inflammation, as well as growth factor and KRAS signaling. ADM is a reversible process and is involved in pancreatic regeneration when pancreatitis resolves [1]. It was shown in different mouse models that in the presence of an oncogenic KRAS mutation, ADM is irreversible and leads to the development of pancreatic intraepithelial neoplasia (PanIN), which can further develop into pancreatic ductal adenocarcinoma (PDAC) $[4,5,12,27]$. While the factors that induce ADM are increasingly identified, it remains unclear whether they utilize a common signaling pathway to promote their effects on acinar cell plasticity.

In this study, we show that the generation of hydrogen peroxide is an event that occurs downstream of all the tested inducers of ADM (Figures 1 and 2). Moreover, ADM can be induced via extrinsic hydrogen peroxide (Figure $3 A, B$ ). We also show that mitochondriallytargeted catalase and not inhibition of NADPH oxidase can block ADM, suggesting that mitochondria are the origin of ROS signaling (Figure 3C,D; Supplemental Figure S3 and [15]). This seems to be different to fully developed pancreatic cancer, where KRAS-induced NADPH oxidase signaling increases cellular superoxide and hydrogen peroxide levels [28]. In our previous work, we showed that during the ADM process, mutant KRAS induces mitochondrial dysfunction, which results in increased mitochondrial oxidative stress [15,29]. Our data presented here expand this finding to other inducers of ADM, such as TGF $\alpha$ and M-CM. Protein Kinase D1 (PKD1) can be activated by mitochondrial oxidative stress [16-18]. During the ADM process, PKD1 is activated downstream of EGFR and mutant KRAS [14], and here we show that it is also downstream of M-CM and CCL5 (Figure 4). Moreover, extrinsic hydrogen peroxide-induced ADM was efficiently blocked when PKD1 was inhibited (Figure 4E).

Pancreatic acinar-to-ductal metaplasia was previously linked to the activation of the transcription factors Notch [30,31], NF-kB [10], STAT3 [32] and NFAT1/4 [33,34], all of which are directly or indirectly activated by PKD1 in acinar cells. For example, it was shown that downstream of KRAS signaling (mutant KRAS and EGFR-KRAS), PKD1 regulates ADM via the activation of Notch [14]. This is most likely achieved through the activation of MMP7, which can cleave Notch to generate the activated version NICD (Notch intracellular domain) [31]. Consequently, Notch target genes such as Hey1, Hes1, Sox9 and pancreatic and duodenal homeobox-1 ( $P d x 1)$, which are increasingly expressed in cells that undergo ADM [31,35-37], are all induced by active PKD1 [14]. Another target transcription factor for PKD1 is NF- $\mathrm{kB}$, which in acinar cells upregulates the expression of EGFR and its ligands [15] and possibly contributes to signal amplification via EGFR signaling to induce STAT3 and NFAT1/4 $[33,34]$. STAT3 has been shown to regulate ADM downstream of YAP1 [1,32], which is also regulated via PKD1 [38]. However, it should be noted that other factors, such as Myc and KLF4, have been shown to be required for the ADM process in mice $[39,40]$ and that the relation and role of PKD1 in their activation is not yet established.

A pathological progression model for PDAC suggests that it originates in ADM progenitor cells that give rise to PanIN $[1,5,41]$. Since our data show that ROS-PKD1 signaling is a central driver of the ADM process, the pharmacologic inhibition of this signaling pathway may be an effective strategy to prevent early oncogenic changes. Options to reduce ADM events in vivo could include the use of antioxidants or PKD1 inhibitors. While antioxidant strategies to target ROS homeostasis have been tested for PDAC with mixed results (discussed in [42]), the inhibition of PKD1 as a downstream target for ROS may be a more-specific option. However, at this point, no PKD inhibitors have been developed that can be clinically used. Further, inhibitor specificity for the PKD1 isoform may be important, since other PKD isoforms, such as PKD3, can regulate critical acinar cell functions, including amylase secretion [43].

\section{Conclusions}

Pancreatic acinar-to-ductal metaplasia is a de-differentiation process and cells that undergo $\mathrm{ADM}$ can initiate the formation of pancreatic precancerous lesions when an onco- 
genic mutation of KRAS is present (reviewed in [1,5]). Multiple inducers of ADM have been identified [7-11], but so far, no common underlying signaling mechanism has been described. In this study, we identify the generation of mitochondrial ROS and ROS-mediated activation of PKD1 as a common signaling pathway for the inducers of ADM, including CCL5, TNF, EGFR and KRAS. Since PKD1 links to most transcription factors crucial for the acinar cell de-differentiation process, this kinase may be a critical and targetable component to develop strategies to prevent the development of pancreatic cancer.

Supplementary Materials: The following supporting information can be downloaded at: https: / / www.mdpi.com/article/10.3390/antiox11010137/s1. Figure S1: TGF $\alpha$ induces RNA expression related to ADM; and TGF $\alpha$-induced ADM is blocked by NAC; Figure S2: Macrophage-conditioned media induces ADM; Figure S3: The knockdown of p22 phox does not affect ADM; Figure S4: Role of PKD1 in ADM; Figure S5: TGF $\alpha$ - and CCL5-induced ADM is inhibited by super-dominant IkB $\alpha$.

Author Contributions: G.-Y.L., H.R.D. and P.S. conceived and designed the experiments. G.-Y.L. and H.R.D. performed the experiments. G.-Y.L., H.R.D. and P.S. analyzed the data: P.S. wrote the paper. All authors have read and agreed to the published version of the manuscript.

Funding: This work was supported by NIH grants CA200572, CA229560 and CA244167 to PS. The content is solely the responsibility of the authors and does not necessarily represent the official views of the National Cancer Institute or the National Institutes of Health. The funders had no role in study design, data collection and analysis, the decision to publish, or the preparation of the manuscript.

Institutional Review Board Statement: The Institutional Animal Care and Use Committee (IACUC) at Mayo Clinic approved the animal experiments under protocol A00004882.

Informed Consent Statement: Not applicable.

Data Availability Statement: Data are contained in the article and supplementary materials.

Acknowledgments: We thank Q. Jane Wang (University of Pittsburgh) for providing the PKD inhibitor kb-NB-142-70, Yan Asmann (Associate Professor of Biomedical Informatics, Mayo Clinic) for advice on the statistical analyses, Alicia Fleming-Martinez for editing the manuscript and our colleagues in the Storz laboratory and the Department for Cancer Biology for helpful discussions.

Conflicts of Interest: The authors declare no conflict of interest.

$\begin{array}{ll}\text { Abbreviations } \\ \text { ADM } & \begin{array}{l}\text { acinar-to-ductal metaplasia } \\ \text { chemokine (C-C motif) ligand } 5\end{array} \\ \text { CCL5 } & \begin{array}{l}\text { cytokeratin 19 } \\ \text { CK19 }\end{array} \\ \text { EGF } & \text { epidermal growth factor } \\ \text { EGFR } & \text { epidermal growth factor receptor } \\ \text { M-CM } & \text { macrophage-conditioned media } \\ \text { NF- } \mathrm{B} \text { n } & \text { nuclear factor-kB } \\ \text { PanIN } & \text { pancreatic intraepithelial neoplasia } \\ \text { PDAC } & \text { pancreatic ductal adenocarcinoma } \\ \text { PKD1 } & \text { Protein Kinase D1 } \\ \text { ROS } & \text { reactive oxygen species } \\ \text { TGF } \alpha & \text { transforming growth factor } \alpha \\ \text { TNF } & \text { tumor necrosis factor }\end{array}$

\section{References}

1. Storz, P. Acinar cell plasticity and development of pancreatic ductal adenocarcinoma. Nat. Rev. Gastroenterol. Hepatol. 2017, 14, 296-304. [CrossRef] [PubMed]

2. Pin, C.; Rukstalis, J.M.; Johnson, C.; Konieczny, S.F. The bHLH transcription factor Mist1 is required to maintain exocrine pancreas cell organization and acinar cell identity. J. Cell Biol. 2001, 155, 519-530. [CrossRef]

3. Strobel, O.; Dor, Y.; Alsina, J.; Stirman, A.; Lauwers, G.; Trainor, A.; Castillo, C.F.; Warshaw, A.L.; Thayer, S.P. In Vivo Lineage Tracing Defines the Role of Acinar-to-Ductal Transdifferentiation in Inflammatory Ductal Metaplasia. Gastroenterology 2007, 133, 1999-2009. [CrossRef] 
4. Morris, J.P., IV; Cano, D.A.; Sekine, S.; Wang, S.C.; Hebrok, M. Beta-catenin blocks Kras-dependent reprogramming of acini into pancreatic cancer precursor lesions in mice. J. Clin. Investig. 2010, 120, 508-520. [CrossRef] [PubMed]

5. Storz, P.; Crawford, H.C. Carcinogenesis of Pancreatic Ductal Adenocarcinoma. Gastroenterology 2020, 158, 2072-2081. [CrossRef] [PubMed]

6. Collins, M.A.; Yan, W.; Sebolt-Leopold, J.S.; di Magliano, M.P. MAPK Signaling Is Required for Dedifferentiation of Acinar Cells and Development of Pancreatic Intraepithelial Neoplasia in Mice. Gastroenterology 2014, 146, 822-834.e7. [CrossRef] [PubMed]

7. Crawford, H.C.; Scoggins, C.R.; Washington, M.K.; Matrisian, L.M.; Leach, S.D. Matrix metalloproteinase-7 is expressed by pancreatic cancer precursors and regulates acinar-to-ductal metaplasia in exocrine pancreas. J. Clin. Investig. 2002, 109, 1437-1444. [CrossRef] [PubMed]

8. De Lisle, R.C.; Logsdon, C.D. Pancreatic acinar cells in culture: Expression of acinar and ductal antigens in a growth-related manner. Eur. J. Cell Biol. 1990, 51, 64-75.

9. Esni, F.; Miyamoto, Y.; Leach, S.D.; Ghosh, B. Primary explant cultures of adult and embryonic pancreas. Methods Mol. Med. 2005, 103, 259-271.

10. Liou, G.-Y.; Döppler, H.; Necela, B.; Krishna, M.; Crawford, H.C.; Raimondo, M.; Storz, P. Macrophage-secreted cytokines drive pancreatic acinar-to-ductal metaplasia through NF-kB and MMPs. J. Cell Biol. 2013, 202, 563-577. [CrossRef]

11. Means, A.L.; Meszoely, I.M.; Suzuki, K.; Miyamoto, Y.; Rustgi, A.K.; Coffey, R.J., Jr.; Wright, C.V.E.; Stoffers, D.A.; Leach, S.D. Pancreatic epithelial plasticity mediated by acinar cell transdifferentiation and generation of nestin-positive intermediates. Development 2005, 132, 3767-3776. [CrossRef] [PubMed]

12. Hingorani, S.; Petricoin, E.F.; Maitra, A.; Rajapakse, V.; King, C.; Jacobetz, M.A.; Ross, S.; Conrads, T.P.; Veenstra, T.D.; Hitt B.A.; et al. Preinvasive and invasive ductal pancreatic cancer and its early detection in the mouse. Cancer Cell 2003, 4, 437-450. [CrossRef]

13. Sandgren, E.P.; Luetteke, N.C.; Palmiter, R.D.; Brinster, R.L.; Lee, D.C. Overexpression of TGF $\alpha$ in transgenic mice: Induction of epithelial hyperplasia, pancreatic metaplasia, and carcinoma of the breast. Cell 1990, 61, 1121-1135. [CrossRef]

14. Liou, G.Y.; Doppler, H.; Braun, U.B.; Panayiotou, R.; Scotti Buzhardt, M.; Radisky, D.C.; Crawford, H.C.; Fields, A.P.; Murray, N.R.; Wang, Q.J.; et al. Protein kinase D1 drives pancreatic acinar cell reprogramming and progression to intraepithelial neoplasia. Nat. Commun. 2015, 6, 6200. [CrossRef] [PubMed]

15. Liou, G.-Y.; Döppler, H.; DelGiorno, K.E.; Zhang, L.; Leitges, M.; Crawford, H.C.; Murphy, M.P.; Storz, P. Mutant KRas-Induced Mitochondrial Oxidative Stress in Acinar Cells Upregulates EGFR Signaling to Drive Formation of Pancreatic Precancerous Lesions. Cell Rep. 2016, 14, 2325-2336. [CrossRef] [PubMed]

16. Storz, P.; Doppler, H.; Toker, A. Protein Kinase C $\delta$ Selectively Regulates Protein Kinase D-Dependent Activation of NF- $k B$ in Oxidative Stress Signaling. Mol. Cell. Biol. 2004, 24, 2614-2626. [CrossRef]

17. Storz, P.; Doppler, H.; Toker, A. Protein Kinase D Mediates Mitochondrion-to-Nucleus Signaling and Detoxification from Mitochondrial Reactive Oxygen Species. Mol. Cell. Biol. 2005, 25, 8520-8530. [CrossRef]

18. Storz, P.; Toker, A. Protein kinase D mediates a stress-induced NF-kappaB activation and survival pathway. EMBO J. 2003, 22, 109-120. [CrossRef] [PubMed]

19. Avila, J.L.; Troutman, S.; Durham, A.; Kissil, J.L. Notch1 Is Not Required for Acinar-to-Ductal Metaplasia in a Model of Kras-Induced Pancreatic Ductal Adenocarcinoma. PLoS ONE 2012, 7, e52133. [CrossRef]

20. Sharlow, E.R.; Mustata Wilson, G.; Close, D.; Leimgruber, S.; Tandon, M.; Reed, R.B.; Shun, T.Y.; Wang, Q.J.; Wipf, P.; Lazo, J.S. Discovery of Diverse Small Molecule Chemotypes with Cell-Based PKD1 Inhibitory Activity. PLoS ONE 2011, 6, e25134 [CrossRef]

21. Martinez, A.K.F.; Döppler, H.R.; Bastea, L.I.; Edenfield, B.; Patel, T.; Leitges, M.; Liou, G.-Y.; Storz, P. Dysfunctional EGFR and oxidative stress-induced PKD1 signaling drive formation of DCLK1+ pancreatic stem cells. iScience 2021, 24, 102019. [CrossRef]

22. Pandey, V.; Fleming-Martinez, A.; Bastea, L.; Doeppler, H.R.; Eisenhauer, J.; Le, T.; Edenfield, B.; Storz, P. CXCL10/CXCR3 signaling contributes to an inflammatory microenvironment and its blockade enhances progression of murine pancreatic precancerous lesions. eLife 2021, 10, e60646. [CrossRef] [PubMed]

23. Ardito, C.M.; Grüner, B.M.; Takeuchi, K.K.; Lubeseder-Martellato, C.; Teichmann, N.; Mazur, P.K.; Delgiorno, K.E.; Carpen-ter, E.S.; Halbrook, C.J.; Hall, J.C.; et al. EGF Receptor Is Required for KRAS-Induced Pancreatic Tumorigenesis. Cancer Cell 2012, 22, 304-317. [CrossRef] [PubMed]

24. Martinez, A.K.F.; Storz, P. Mimicking and Manipulating Pancreatic Acinar-to-Ductal Metaplasia in 3-dimensional Cell Culture. J. Vis. Exp. 2019, 144, e59096. [CrossRef]

25. Baker, K.; Marcus, C.B.; Huffman, K.; Kruk, H.; Malfroy, B.; Doctrow, S.R. Synthetic combined superoxide dismutase/catalase mimetics are protective as a delayed treatment in a rat stroke model: A key role for reactive oxygen species in ischemic brain injury. J. Pharmacol. Exp. Ther. 1998, 284, 215-221. [PubMed]

26. Paoli, C.; Carrer, A. Organotypic Culture of Acinar Cells for the Study of Pancreatic Cancer Initiation. Cancers 2020, $12,2606$. [CrossRef]

27. Carriere, C.; Seeley, E.S.; Goetze, T.; Longnecker, D.S.; Korc, M. The Nestin progenitor lineage is the compartment of origin for pancreatic intraepithelial neoplasia. Proc. Natl. Acad. Sci. USA 2007, 104, 4437-4442. [CrossRef]

28. Wang, P.; Sun, Y.-C.; Lu, W.-H.; Huang, P.; Hu, Y. Selective killing of K-ras-transformed pancreatic cancer cells by targeting NAD(P)H oxidase. Chin. J. Cancer 2015, 34, 166-176. [CrossRef] [PubMed] 
29. Weinberg, F.; Hamanaka, R.; Wheaton, W.W.; Weinberg, S.; Joseph, J.; Lopez, M.; Kalyanaraman, B.; Mutlu, G.M.; Budinger, G.R.S.; Chandel, N.S. Mitochondrial metabolism and ROS generation are essential for Kras-mediated tumorigenicity. Proc. Natl. Acad. Sci. USA 2010, 107, 8788-8793. [CrossRef]

30. Lee, K.M.; Yasuda, H.; Hollingsworth, M.A.; Ouellette, M.M. Notch2-positive progenitors with the intrinsic ability to give rise to pancreatic ductal cells. Lab. Investig. 2005, 85, 1003-1012. [CrossRef]

31. Sawey, E.; Johnson, J.A.; Crawford, H.C. Matrix metalloproteinase 7 controls pancreatic acinar cell transdifferentiation by activating the Notch signaling pathway. Proc. Natl. Acad. Sci. USA 2007, 104, 19327-19332. [CrossRef] [PubMed]

32. Gruber, R.; Panayiotou, R.; Nye, E.; Spencer-Dene, B.; Stamp, G.; Behrens, A. YAP1 and TAZ Control Pancreatic Cancer Initiation in Mice by Direct Up-regulation of JAK-STAT3 Signaling. Gastroenterology 2016, 151, 526-539. [CrossRef]

33. Chen, N.-M.; Singh, G.; Koenig, A.; Liou, G.-Y.; Storz, P.; Zhang, J.-S.; Regul, L.; Nagarajan, S.; Kühnemuth, B.; Johnsen, S.A.; et al. NFATc1 Links EGFR Signaling to Induction of Sox9 Transcription and Acinar-Ductal Transdifferentiation in the Pancreas. Gastroenterology 2015, 148, 1024-1034.e9. [CrossRef] [PubMed]

34. Hessmann, E.; Zhang, J.-S.; Chen, N.-M.; Hasselluhn, M.C.; Liou, G.-Y.; Storz, P.; Ellenrieder, V.; Billadeau, D.D.; Koenig, A. NFATc4 RegulatesSox9Gene Expression in Acinar Cell Plasticity and Pancreatic Cancer Initiation. Stem Cells Int. 2016, 2016, 5272498. [CrossRef]

35. Hosokawa, S.; Furuyama, K.; Horiguchi, M.; Aoyama, Y.; Tsuboi, K.; Sakikubo, M.; Goto, T.; Hirata, K.; Tanabe, W.; Nakano, Y.; et al. Impact of Sox9 Dosage and Hes1-mediated Notch Signaling in Controlling the Plasticity of Adult Pancreatic Duct Cells in Mice. Sci. Rep. 2015, 5, 8518. [CrossRef]

36. Shroff, S.; Rashid, A.; Wang, H.; Katz, M.H.; Abbruzzese, J.L.; Fleming, J.B.; Wang, H. SOX9: A useful marker for pancreatic ductal lineage of pancreatic neoplasms. Hum. Pathol. 2014, 45, 456-463. [CrossRef]

37. Song, S.Y.; Gannon, M.; Washington, M.K.; Scoggins, C.R.; Meszoely, I.M.; Goldenring, J.R.; Marino, C.R.; Sandgren, E.P.; Coffey, R.J., Jr.; Wright, C.V.; et al. Expansion of Pdx1-expressing pancreatic epithelium and islet neogenesis in transgenic mice overexpressing transforming growth factor alpha. Gastroenterology 1999, 117, 1416-1426. [CrossRef]

38. Wang, J.; Sinnett-Smith, J.; Stevens, J.V.; Young, S.H.; Rozengurt, E. Biphasic Regulation of Yes-associated Protein (YAP) Cellular Localization, Phosphorylation, and Activity by G Protein-coupled Receptor Agonists in Intestinal Epithelial Cells: A Novel Role for Protein Kinase D (PKD). J. Biol. Chem. 2016, 291, 17988-18005. [CrossRef]

39. Grippo, P.J.; Sandgren, E.P. Acinar-to-ductal metaplasia accompanies c-myc-induced exocrine pancreatic cancer progression in transgenic rodents. Int. J. Cancer 2012, 131, 1243-1248. [CrossRef]

40. Wei, D.; Wang, L.; Yan, Y.; Jia, Z.; Gagea, M.; Li, Z.; Zuo, X.; Kong, X.; Huang, S.; Xie, K. KLF4 Is Essential for Induction of Cellular Identity Change and Acinar-to-Ductal Reprogramming during Early Pancreatic Carcinogenesis. Cancer Cell 2016, 29, 324-338. [CrossRef]

41. Hruban, R.H.; Adsay, N.V.; Albores-Saavedra, J.; Compton, C.; Garrett, E.S.; Goodman, S.N.; Kern, S.E.; Klimstra, D.S.; Kloppel, G.; Longnecker, D.S.; et al. Pancreatic intraepithelial neoplasia: A new nomenclature and classification system for pancreatic duct lesions. Am. J. Surg. Pathol. 2001, 25, 579-586. [CrossRef] [PubMed]

42. Durand, N.; Storz, P. Targeting reactive oxygen species in development and progression of pancreatic cancer. Expert Rev. Anticancer Ther. 2017, 17, 19-31. [CrossRef] [PubMed]

43. Chen, L.A.; Li, J.; Silva, S.R.; Jackson, L.N.; Zhou, Y.; Watanabe, H.; Ives, K.L.; Hellmich, M.R.; Evers, B.M. PKD3 Is the Predominant Protein Kinase D Isoform in Mouse Exocrine Pancreas and Promotes Hormone-induced Amylase Secretion. J. Biol. Chem. 2009, 284, 2459-2471. [CrossRef] [PubMed] 